\title{
EDITORIAL
}

\section{Recent advances in bone marrow biopsy pathology}

\author{
Jon van der Walt
}

Received: 24 April 2009/Accepted: 28 April 2009/Published online: 14 May 2009

(C) Springer-Verlag 2009

Advances in bone marrow pathology continue apace in 2009. The following publications are a personal selection of highlights which are especially applicable to bone marrow biopsies (BMB).

\section{Myeloproliferative neoplasms}

The revised 2008 World Health Organization (WHO) classification established a new category of "acute myeloid leukemia (AML) with myelodysplasia-related changes" (AML-MRC). Weinberg et al. [1] evaluated the clinical, pathologic, cytogenetic, and molecular features of 100 AML patients using the 2008 WHO criteria. Patients underwent genetic screening for NPM1, FLT3-ITD, FLT3-D835, and CEBPA mutations. Compared with patients with AML, not otherwise specified, patients with AML-MRC were significantly older, presented with a lower hemoglobin, more frequently expressed CD14, and exhibited a decreased frequency of CEBPA mutations. Multivariate analysis indicated that patients with AML-MRC had a significantly worse overall survival, progression-free survival, and complete response compared with AML, not otherwise specified. The authors conclude that their data support the clinical, morphologic, and cytogenetic criteria for this 2008 WHO AML category.

Therapy-related myeloid neoplasm (t-MN) is the term recently proposed by the World Health Organization to cover the spectrum of disorders previously described as therapy-related myelodysplastic syndrome (t-MDS) or

J. van der Walt $(\square)$

Department of Histopathology, St. Thomas' Hospital,

Westminster Bridge Road,

London SE1 7EH, UK

e-mail: Jon.vanderwalt $@$ kcl.ac.uk therapy-related acute myeloid leukemia (t-AML). Larson [2] has reviewed the current views on this new category and the diagnostic highlights are summarized here.

$\mathrm{t}-\mathrm{MN}$ is recognized as a clinical syndrome occurring as a late complication following cytotoxic therapy. The term therapy-related leukemia is based on a patient's history of exposure to cytotoxic agents and implies a causal relationship although the precise mechanism remains to be proven. These neoplasms are thought to be the direct consequence of mutational events induced by cytotoxic therapy or via the selection of a myeloid clone with a markedly elevated risk for mutational events. Several distinct clinical and cytogenetic subtypes of t-MN are recognized and closely associated with the nature of the preceding treatment. Therapy-related disease may develop from a few months to several years after therapy, depending both on the cumulative dose or dose intensity of the preceding cytotoxic therapy and exposure to specific agents. The majority of patients have clonal chromosome abnormalities in their bone marrow cells at diagnosis.

A spectrum of morphological abnormalities is observed. There is a continuum in the percentage of marrow blasts from t-MDS to overt acute leukemia and rapid progression from the former to the latter. These observations support the WHO categorization as a single disorder. The clinical course is typically progressive and relatively resistant to conventional therapies used for leukemias arising de novo.

The classical form of therapy-related leukemia follows treatment with alkylating agents and/or radiation therapy. The blood and bone marrow findings resemble those seen in primary MDS, although the degree of dysgranulopoiesis and dysmegakaryocytopoiesis is typically greater. Marked dysplastic changes are observed in all three cell lines. Mild to marked reticulin fibrosis may be present. Complex clonal chromosomal abnormalities are identified in most cases. 
Loss of part or all of chromosomes 5 and/or 7 is the characteristic findings and has been reported in over $90 \%$ of cases. The most common single abnormality is monosomy 7 , followed in frequency by deletion of the long arm of chromosome 5 [del(5q)] and by monosomy 5 [2].

Therapy-related leukemia secondary to topoisomerase II inhibitors typically result in translocations involving the MLL gene on chromosome 11, band q23, and, less commonly, the AML1 gene on chromosome 21, band q22. In contrast to classic t-MN, these leukemias have a much shorter latency period and a preceding MDS is not common.

Neoplasia is traditionally thought to arise from a single cell. However, recently, it has been proposed that divergent clones may either be derived from the original clone or represent distinct and unrelated clones.

Beer et al. [3] looked for clonal diversity in patients with a myeloproliferative neoplasm (MPN) associated with more than one acquired genetic lesion. A tyrosine kinase mutation and a cytogenetic lesion were found in the same clone in six of seven patients. By contrast, the genetic lesions were present in separate clones in all six patients with two tyrosine kinase pathway mutations. In two patients, the clones were genetically unrelated by $\mathrm{X}$ chromosome inactivation studies. This study demonstrated clonal diversity in a subset of patients with early-stage hematopoietic malignancy and showed, for the first time, that such clones may arise independently. Perhaps findings such as these may explain some of the difficulties in the morphological classification of MPN.

Acute myeloid leukemia carrying cytoplasmic mutated nucleophosmin (NPMc+AML) and blastic plasmacytoid dendritic cell neoplasm (BPDCN) were included as new entities in the revised WHO classification of myeloid neoplasms. These conditions may show clinical and pathological overlapping features including leukemia and skin involvement and expression of macrophage markers. Facchetti et al. [4] studied both entities in BMB and showed that aberrant cytoplasmic dislocation of nucleophosmin - the immunohistochemical surrogate for NPM1 mutations - allows the two entities to be genetically separated. Nucleophosmin was consistently cytoplasmic in NPMc+AML (due to the presence of NPM1 mutations), while it was nucleus-restricted (predictive of a germ line NPM1 gene) in BPDCN. Cytoplasmic nucleophosmin is therefore a useful marker for distinguishing NPMc+ AML from BPDCN and should be included in immunopanels for the differential diagnosis of these disorders.

\section{The myelodysplastic/myeloproliferative neoplasms}

The WHO diagnostic criteria for chronic myelomonocytic leukemia (CMML) are mainly based on the peripheral blood and bone marrow aspirate. According to definition, the presence of peripheral blood monocytosis is essential for the diagnosis of CMML. Human peripheral blood monocytes are distributed between a major CD14+ CD16- population representing $90-95 \%$ of total blood monocytes and a minor CD14 low CD16+ population [5]. CD14 is considered to be a good marker of the major monocyte population and can be now evaluated on paraffin-embedded tissue [5]. Although flow cytometry has demonstrated a moderate increase of CD14+ cells in CMML, the level and pattern of monocytic proliferation in the BMB of patients have not been clearly established.

Qubajal et al. [5] immunostained 15 paraffin-embedded cases of CMML, including two new antibodies staining for CD14 and CD16. CD68 (KP1), CD68 (PG-M1), and CD163 were not differentially expressed between CMML and chronic myelogenous leukemia (CML). In CMML, there was a significant increase in the number of CD14+ monocytes, comprising dispersed cells in the interstitium, with frequent bilobated nuclei, difficult to differentiate from neutrophils. There was no expansion of CD16+ monocytelike cells. There was, however, a significant decrease in the number of granulocytes expressing CD16, MPO, and CD15 in CMML compared to CML and control BMB, probably related to dysgranulopoiesis. The authors conclude that BMB immunohistochemistry can be helpful in the diagnosis of CMML by identifying both the monocyte expansion with CD14 and the dysgranulopoiesis with CD16.

Both the 2001 and 2008 editions of the WHO classification of hematopoietic neoplasms include refractory anemia with ring sideroblasts and thrombocytosis (RARS-T) as distinct diagnostic category, describing patients who have both $\geq 15 \%$ ring sideroblasts and a sustained elevated platelet count. However, it has recently become apparent that patients fulfilling the WHO diagnostic criteria for RARS-T have clonal JAK2 ${ }^{\mathrm{V} 617 \mathrm{~F}}$ and MPL ${ }^{\mathrm{W} 515}$ mutations similar to the reported rate for essential thrombocythemia (ET).

Wardrop and Steensma [6] have reviewed the historic literature and current molecular evidence on RARS-T and conclude that, given that the provisional classification of RARS-T as a MDS/MPN overlap syndrome, rather than as a form of MPN (i.e., ET), rests principally upon the presence of ring sideroblasts and that these are nonspecific morphological findings, these new molecular results should prompt reconsideration of RARS-T as a distinct category. The authors argue cogently against the continued use of RARS-T as a separate diagnostic category in comparison with a simplifying diagnosis of ET with ring sideroblasts.

\section{Myelodysplastic syndromes}

Hypocellular or hypoplastic acute myeloid leukemia $(\mathrm{H}-$ AML) and hypocellular myelodysplastic syndromes (H- 
MDS) represent a small fraction of myeloid malignancies. Pronounced cytopenia may raise the question of a diagnosis of acquired aplastic anemia (AA) and it may be very difficult to separate these disorders from each other utilizing morphological criteria applied to bone marrow aspirate smears since criteria such as blast counts and degree of morphological dysplasia may overlap. In addition, the marked hypocellularity of bone marrow aspirates render interpretation difficult. Thus, a bone marrow biopsy is critical and necessary to diagnose these variants in all patients, children included [7].

Bennett and Orazi [7] report the conclusions of three workshops held by members of the French-AmericanBritish Cooperative Leukemia Working Group.

The following approach was used:

1. The presence of unequivocal blasts in the peripheral blood is considered indicative of MDS or AML. Because of significant leukopenia, a precise determination of blast percentage is often impossible, but an effort should be made to count at least 100 cells, including lymphocytes.

2. Hypogranular neutrophils or pseudo-Pelger neutrophils are considered indicative of either MDS or AML if more than $10 \%$ are identified. Fewer numbers raise the suspicion but are not felt to be definitive.

3. The presence of $>1 \%$ to $20 \%$ blasts in the marrow aspirates is considered diagnostic of MDS if dysplasia is recognized.

4. Morphological marrow dysplasia of either granulocytes or megakaryocytes is considered as abnormal and inconsistent with AA. Erythroid dysplasia must be moderate to severe, if it is the sole finding (binucleated or trinucleated forms, numerous Howell-Jolly bodies, nuclear budding or bridging).

5. The presence of any abnormal sideroblasts ( $>5$ granules surrounding the nuclear membrane or occupying at least one third of the circumference) is considered as evidence of dyserythropoiesis and excluded a diagnosis of AA.

6. A 1- to 2-cm core biopsy is preferred for all patients being evaluated for a potential diagnosis of either hypoplastic myeloid disorder or aplastic anemia.

7. The presence of two or more clusters of immature precursors (minimum of three blasts/clusters) in the bone marrow biopsy is indicative of either MDS or AML

H-AML proved to be the most contentious diagnosis. The diagnosis of myeloid neoplasms with fatty marrow such $\mathrm{H}$ MDS and H-AML may be very difficult, particularly in cases with a bone marrow cellularity of less than $10 \%$. The established criteria of a $5 \%$ minimal blast percentage in the marrow for MDS and below $1 \%$ in AA require the use of a
500 cell differential count as recommended by the WHO system.

The presence of easily identifiable megakaryocytes within an architecturally disorganized marrow and the presence of reticulin fibrosis favor MDS over aplastic anemia. An important feature of the BMB in cases with fatty marrow and paucicellular aspirates is its ability to identify blasts. The presence of aggregates or clusters of blasts, previously defined as abnormally localized immature myeloid precursor cells (ALIP), is mainly seen in the aggressive MDS subtypes and is associated with a poor prognosis and an increased incidence of progression to acute leukemia. Cases are classified as ALIP positive if at least three aggregates (more than five myeloid precursors) or clusters (three to five myeloid precursors) are identified in a histology section. Immunohistological staining for CD34 is an essential tool for counting blasts in tissue sections. Other antibodies that may be useful include CD117, myeloperoxidase, lysozyme, and CD68. Both an increase in the percentage of CD34-positive cells and a tendency of positive cells to form aggregates have been shown to be useful in distinguishing H-MDS and H-AML from AA.

The diagnosis remains difficult in cases with a bone marrow cellularity of less than $10 \%$ but cytogenetic analysis failures are frequent. Although there is often good correlation between CD34 percentage as determined by flow cytometry and morphology, the percentage of blasts as determined by flow may be influenced by hemodilution and the result from flow cytometry should never be used in lieu of morphological inspection of the peripheral blood film, bone marrow aspirate smears, and bone marrow biopsy. The authors emphasize the value of a multiparameter approach and the necessity for experience in the diagnosis of these related conditions.

\section{Myeloid and lymphoid neoplasms with eosinophilia and abnormalities of PDGFRA, PDGFRB, and FGFR1}

The hypereosinophilic syndromes (HES) encompass a spectrum of diseases that have increased blood eosinophils and tissue damage in common and protean clinical manifestations, involving any organ system but especially the skin [8]. Gleich and Leiferman [8] present a comprehensive review of HES with discussion of the various classifications that characterize patients with marked eosinophilia. A classification is proposed: (1) myeloproliferative HES (chronic eosinophilic leukemia with a unique genetic marker, FIP1L1-PDGFRA, and well controlled by administration of the kinase inhibitor, imatinib, and FIP1L1PDGFRA negative cases of unknown etiology); (2) lymphocytic HES associated with $\mathrm{T}$ cell clones producing 
interleukin 5 which can evolve into lymphoma; (3) undefined HES (benign, complex, and episodic); (4) familial HES; (5) associated HES; (6) overlap HES.

While myeloproliferative and lymphocytic HES are well established and permit elimination of the term idiopathic for these varieties, most HES patients do not fall into these categories and are classified as complex [8].

The identification of myeloproliferative HES depends on identification of clonal eosinophilic myeloid populations, especially those harboring the FIP1L1-PDGFRA mutant gene and identification of lymphocytic HES rests upon recognition of distinct helper T cell subsets (TH1 and TH2) and clonal overgrowth of specific cytokine-producing cells. The value of the BMB is emphasized and the importance of investigation of the involvement of other cell lineages in particular mast cells is highlighted. It is recommended that biopsies should be examined both by conventional microscopy and also by staining with antibodies to eosinophil granule proteins because eosinophil granule protein deposition may occur in the virtual absence of intact eosinophils.

\section{Lymphoid lesions in the bone marrow}

Mantle cell lymphoma (MCL) and chronic lymphocytic leukemia (CLL) are CD5+ small B cell neoplasms (SBCNs) with overlapping features. Flow cytometry is often used to help differentiate CLL from MCL, and a characteristic CLL phenotype is considered essentially diagnostic [9]. Ho et al. [9] analyzed 28 cases of SBCN by flow cytometry and found typical MCL immunophenotypes. However, fluorescence in situ hybridization (FISH) analysis indicated that $57 \%$ had $\mathrm{t}(11 ; 14)$ translocations consistent with MCL, while $32 \%$ lacked $\mathrm{t}(11 ; 14)$ translocations but had other cytogenetic abnormalities commonly found in CLL. The authors found no significant morphologic or immunophenotypic differences between the $t(11 ; 14)$-positive and $t(11 ; 14)$-negative cases. They conclude that many blood-based SBCNs with typical MCL immunophenotypes likely represent cases of phenotypically atypical CLL.

The BMB analysis may thus be crucial in the diagnosis of MCL and atypical CLL and both immunocytochemistry for cyclin D1 and FISH for $\mathrm{t}(11 ; 14)$ should be employed in SBCN of indeterminate type,

Feldman et al. [10] identified recurrent translocations involving the multiple myeloma oncogene $1 /$ interferon regulatory factor 4 (IRF4) locus in peripheral $\mathrm{T}$ cell lymphomas (PTCLs); 169 PTCLs were studied using fluorescence in situ hybridization; 12 cases with IRF4 translocations were identified. Two cases with $\mathrm{t}(6 ; 14)(\mathrm{p} 25$; q11.2) had translocations between IRF4 and the T cell receptor alpha (TCRA) locus. Both were cytotoxic PTCLs, unspecified involving bone marrow and skin. Eight of the remaining ten cases were cutaneous anaplastic large cell lymphomas without TCRA rearrangements. IRF4 translocations exist in myeloma and some B cell lymphomas but had not been reported earlier in PTCLs. Translocations involving IRF4 but not TCRA appear to occur predominantly in cutaneous ALCLs. Cytotoxic PTCL-unspecified involving bone marrow and skin and containing IRF4/TCRA translocations might represent a distinct clinicopathologic entity. This study suggests that immunostaining for MUM1/IRF4 protein should be included in immunopanels for cases of PTCL involving BMB and skin.

Antibody therapy is being employed in an increasing range of disorders. Jiang et al. [11] carried out a study of CD52 (alemtuzumab) expression using flow cytometry in 78 untreated patients diagnosed with mature T/natural killer (NK) cell neoplasms, including 34 adult $\mathrm{T}$ cell leukemia/lymphomas (ATLL), two ALCLs, three angioimmunoblastic $\mathrm{T}$ cell lymphomas (AITL), 16 cutaneous T cell lymphomas (CTCL), four extranodal T/NK cell lymphomas (ENT/NKCL), four hepatosplenic T cell lymphomas (HSTCL), 13 peripheral T cell lymphomas, not otherwise specified (PTCL-NOS) and two T prolymphocytic leukemia (T-PLL). The level of CD52 expression varied widely within each diagnostic category. All AITL, HSTCL, and T-PLL cases were CD52 positive and the frequency of CD52 expression was high in PTCL-NOS, ATLL, and CTCL but expression was low in ALCL and ENT/NKCL suggesting a role for alemtuzumab in treatment of positive cases but not in negative cases. These findings imply an increasing role for immunocytochemistry in the assessment of which cases in a particular lymphoma type are suitable for antibody therapy and hematopathologists will have to add these antibodies to their standard panels for BMB assessment.

Survivin is selectively expressed in most common human cancers and is now viewed as a potent modulator of the cell death/proliferation balance in tumor cells [12]. Romagnoli et al. [12] had previously reported that myeloma cells expressed high levels of survivin protein in correlation with disease progression and that survivin knockdown by RNA interference decreased myeloma cell growth. In this study [12], the authors demonstrated that survivin overexpression promotes the proliferation and survival of human myeloma cells both in vitro and in vivo in the absence of their major growth factor, interleukin 6 . The effect correlated with the downregulation of Bim, a critical BH3-only cell death activator during cytokine deprivation, mainly at transcriptional level. The tight link between survivin and Bim expression was further confirmed in a panel of newly diagnosed patients with myeloma, and BIRC5 was validated as a gene significantly associated with short survival in these patients. The authors conclude that survivin directly contributes to malignant progression of myeloma and that targeting survivin may disrupt the delicate balance controlling cell 
survival and proliferation. Studies such as these promise an expanding role for immunocytochemical analysis of prognostic markers in BMB.

Interphase FISH coupled to cytoplasmic staining of specific immunoglobulin (cIg-FISH) on bone marrow plasma cells has become well established in the initial evaluation of multiple myeloma [13]. However, little has been published on cytogenetic abnormalities in patients with light-chain amyloidosis.

In a study from the Mayo Clinic, Bryce et al. [13] reviewed 56 patients with light-chain amyloidosis who had cIg-FISH performed as part of their routine clinical testing using their standard screening panel employed in multiple myeloma. Seventy percent of patients had abnormal cIgFISH, with the most common abnormalities being $\operatorname{IgH}$ translocations $(48 \%)$-including $\mathrm{t}(11 ; 14)(39 \%)$ and $\mathrm{t}(14 ; 16)$ $(2 \%)$ - and del13/del13q $(30 \%)$. No $t(4 ; 14)$ or deletions of $17 p$ (p53) were observed. Patients with $t(11 ; 14)$ had the lowest levels of clonal plasma cells, and those with del13 had the highest. The risk of death for patients harboring the $\mathrm{t}(11 ; 14)$ translocation was 2.1 , which on multivariate analysis was independent of therapy. The authors concluded that their preliminary data would suggest that cIg-FISH testing is important in patients with light-chain amyloidosis and that $\mathrm{t}(11 ; 14)$ is an adverse prognostic factor in these patients. This study has implications for BMB analysis and indicates that cyclin D1 should be part of the immunopanel chosen to assess amyloidosis cases.

Boveri et al. [14] reported a case of a 50-year-old HIV+, $\mathrm{HBV}+$, and $\mathrm{HCV}+$ male patient who presented with mild asthenia, splenomegaly slowly progressing anemia, granulocytopenia, lymphocytosis, and mild thrombocytopenia. He was not on antiretroviral therapy.

A BMB showed a discrete lymphoid interstitial infiltrate of mature T cells, which were $\mathrm{CD} 1-, \mathrm{CD} 2+, \mathrm{CD} 3+, \mathrm{CD} 5-/+$, CD7+/-, LAT+, CD4-, CD8+, CD57+/-, TIA1+, perforin+, granzyme $\mathrm{B}+, \mathrm{CD} 56^{-}$, and $\mathrm{CD} 25-$ and had an intrasinusoidal distribution. Polymerase chain reaction demonstrated a monoclonal rearrangement of the $\mathrm{T}$ cell receptor (TCR). Cytologic examination of peripheral blood showed an increased number of large lymphocyte with granular cytoplasm, fulfilling the criteria for the diagnosis of $\mathrm{T}$ cell large granular lymphocytic (T-LGL) leukemia.

The authors note that sinusoidal infiltration is often considered specific for different lymphoma subtypes, either of B cell (splenic marginal zone lymphoma, intravascular large B cell lymphoma) or T cell origin (T-LGL leukemia, hepatosplenic $\mathrm{T}$ cell lymphoma), and has never been described in reactive $\mathrm{T}$ cell proliferations, including reactive expansions of T-LGL. The authors comment that CD8+ LGL can occur during HIV infection and about one third of these subjects carry a monoclonal TCR rearrangement, although without hepatosplenomegaly or a clinically ma- lignant course. HBV is also a known cause of CD8+ lymphocytosis. This short report serves as a reminder that lymphocytosis in HIV infection is not always reactive and deserves thorough investigation.

Persistent polyclonal B cell lymphocytosis (PPBL) is a rare chronic lymphoid hyperplasia characterized by a benign clinical course and a typical clinical setting in middle-aged female smokers [15]. Del Giudice et al. [15] reported five cases of PPBL with progressive splenomegaly. Splenectomies were performed in three cases. BCL2/IGH rearrangements were found in three cases: HLA-DRB $1 * 07$ in all. BMB showed a moderate lymphoid infiltrate with intrasinusoidal distribution resembling a splenic marginal zone lymphoma. Splenic white pulp revealed an enlargement of the marginal zone area: red pulp was infiltrated by the same lymphocytes engulfing the sinuses. Splenic and BMB B lymphocytes were $\mathrm{CD} 79 \mathrm{a}+, \mathrm{CD} 20+, \mathrm{IgM}+, \mathrm{IgD}+$, bcl-2+, CD27+, DBA.44-, CD31-, and polyclonal by immunophenotype/polymerase chain reaction.

The authors conclude that PPBL features an expansion of splenic marginal zone B lymphocytes, which infiltrate $\mathrm{BM}$ sinusoids and circulate in the blood with no evidence of clonality, even in cases with progressive splenomegaly. This paper demonstrates the importance of establishing monoclonality in cases in which a diagnosis of splenic marginal zone lymphoma is entertained in a BMB and of obtaining the relevant clinical history.

\section{Secondary neoplasms}

Evaluation of the BMB is accepted as essential for the accurate staging and follow-up of patients with neuroblastoma. Krishnan et al. [16] evaluated the use of routine immunohistochemical analysis of BMB in neuroblastoma cases. Immunostaining for synaptophysin, chromogranin, and $\beta$-catenin on otherwise histologically negative BMB identified isolated tumor cells (ITCs) in $9.1 \%, 5.0 \%$, and $10.0 \%$ of 220 biopsy specimens, respectively. Overall survival however was not significantly different between patients with and without ITCs. Of the immunohistochemical markers evaluated, $\beta$-catenin showed the greatest sensitivity for identifying ITCs in the bone marrow and showed reactivity in primary tumor samples. $\beta$-catenin appears to a sensitive immunohistochemical marker of primary and secondary neuroblastoma and should thus be used in the routine assessment of BMB in the evaluation of neuroblastoma.

\section{Infectious and inflammatory disorders}

The diagnosis and management of immune thrombocytopenic purpura (ITP) are hampered by a lack of consensus on 
standardized critical definitions, outcome criteria, and terminology. Rodeghiero et al. [17] report the conclusions of an International Working Group of recognized expert clinicians (the Vicenza Consensus Conference) in defining standard terminology and definitions for primary and secondary ITP, different phases of disease and for the grading of severity.

The panel preferred "immune," to "idiopathic," to emphasize the immune-mediated mechanism of the disease and to allow a division into "primary" (as opposed to idiopathic) to indicate the absence of any obvious initiating and/or underlying cause and "secondary immune thrombocytopenia" (secondary ITP) to include all forms of immunemediated thrombocytopenias except primary ITP. The term "purpura" was felt inappropriate because bleeding symptoms are generally absent or minimal. The acronym ITP is however preserved recognizing its time-honored use and facilitating literature searches. A platelet count less than $100 \times 10^{9}$ per liter was preferred to the more commonly used level of less than $150 \times 10^{9}$ per liter.

Secondary ITP includes cases of thrombocytopenias that are due to an underlying disease or to drug exposure. Other examples include fetal and neonatal alloimmune thrombocytopenic purpura, posttransfusion purpura, systemic lupus erythematosus (SLE)-associated, HIV-associated, "druginduced," e.g., "secondary ITP (quinine-induced)" and "heparin-induced thrombocytopenia". The term "newly diagnosed ITP" was recommended for all cases at diagnosis, "persistent ITP," for patients with ITP lasting between 3 and 12 months from diagnosis, including patients not achieving spontaneous remission or not maintaining their response after stopping treatment and "chronic ITP" for patients with ITP lasting for more than 12 months. "Severe" ITP should be used only in patients who have clinically relevant bleeding.

Hematopathologists have a role in the diagnosis of ITP particularly in the exclusion of myelodysplasia. Both SLE and HIV infection with thrombocytopenia have suggestive morphological features in the BMB which may provide the first evidence of these diseases. This review provides a useful clarification and standardization of terminology which is relevant to the reporting of BMB in ITP cases.

Conflict of Interest The author declares no conflict of interest.

\section{References}

1. Weinberg OK, Seetharam M, Ren L, Seo K, Ma L, Merker JD, Gotlib J, Zehnder JL, Arber DA (2009) Clinical characterization of acute myeloid leukemia with myelodysplasia-related changes as defined by the 2008 WHO classification system. Blood 113:1906-1908

2. Larson R (2009) Therapy-related myeloid neoplasms. Haematologica 94:454-459
3. Beer PA, Jones AV, Bench AJ, Goday-Fernandez A, Boyd EM, Vaghela KJ, Erber WN, Odeh B, Wright C, McMullin MF, Jonathan Cullis J, Huntly BJP, Harrison C, Cross NCP, Green AR (2009) Clonal diversity in the myeloproliferative neoplasms: independent origins of genetically distinct clones. B J of Haematol 144:904-908

4. Facchetti F, Pileri SA, Agostinelli C, Martelli MP, Paulli M, Venditti A, Martelli MF, Falini B (2009) Cytoplasmic nucleophosmin is not detected in blastic plasmacytoid dendritic cell neoplasm. Haematologica 94:285-288

5. Qubaja M, Marmey B, Le Tourneau A, Haiat S, Cazals-Hatem D, Fabiani B, Diebold J, Marie J-P, Audouin J, Geissmann F, Molina TJ (2009) The detection of CD14 and CD16 in paraffin-embedded bone marrow biopsies is useful for the diagnosis of chronic myelomonocytic leukaemia. Virchows Arch 454:411-419

6. Wardrop D, Steensma DP (2008) Is refractory anaemia with ring sideroblasts and thrombocytosis (RARS-T) a necessary or useful diagnostic category? B J Haematol 144:809-817

7. Bennett JM, Orazi A (2009) Diagnostic criteria to distinguish hypocellular acute myeloid leukemia from hypocellular myelodysplastic syndromes and aplastic anemia: recommendations for a standardized approach. Haematologica Vol 94:264-268

8. Gleich GJ, Leiferman KM (2009) Review: the hypereosinophilic syndromes: current concepts and treatments. B J Haematol 145:271-285

9. Ho AK, Hill S, Preobrazhensky SN, Miller ME, Chen Z, Bahler DW (2009) Small B-cell neoplasms with typical mantle cell lymphoma immunophenotypes often include chronic lymphocytic leukemias. Am J Clin Pathol 131:27-32

10. Feldman AL, Law M, Remstein ED, Macon WR, Erickson LA, Grogg KL, Kurtin PJ, Dogan A (2009) Recurrent translocations involving the IRF4 oncogene locus in peripheral T-cell lymphomas. Leukemia 23:574-580

11. Jiang L, Yuan CM, Hubacheck J, Janik JE, Wilson W, Morris JC, Jasper GA, Stetler-Stevenson M (2009) Variable CD52 expression in mature $\mathrm{T}$ cell and $\mathrm{NK}$ cell malignancies: implications for alemtuzumab therapy. B J Haematol 145:173-179

12. Romagnoli M, Sèveno C, Wuillëme-Toumi S, Amio M, Bataille R, Minvielle S, Barillè-Nion S (2009) The imbalance between Survivin and Bim mediates tumour growth and correlates with poor survival in patients with multiple myeloma. B J Haematol 145:180-189

13. Bryce AH, Ketterling RP, Gertz MA, Lacy M, Knudson RA, Zeldenrust S, Kumar S, Hayman S, Buadi F, Kyle RA, Greipp PR, Lust JA, Russell S, Rajkumar SV, Fonseca R, Dispenzieri A (2009) Translocation $t(11 ; 14)$ and survival of patients with light chain (AL) amyloidosis. Haematologica 94:380-386

14. Boveri E, Riboni R, Antico P, Malacrida A, Pastorini A (2009) Letter to the Editor: CD3+ T large granular lymphocyte leukaemia in a $\mathrm{HIV}+, \mathrm{HCV}+, \mathrm{HBV}+$ patient. Virchows Arch 454:349-351

15. Del Giudice I, Pileri SA, Rossi M, Sabattini E, Campidelli C, Della Starza I, De Propris MS, Mancini F, Perrone MP, Gesuiti P, Armiento D, Quattrocchi L, Tafuri A, Amendola A, Mauro FR, Guarini A, Fo R (2009) Histopathological and molecular features of persistent polyclonal B-cell lymphocytosis (PPBL) with progressive splenomegaly. B J Haematol 144:726-731

16. Krishnan C, Twist CJ, Fu T, Arber D (2009) Detection of isolated tumor cells in neuroblastoma by immunohistochemical analysis in bone marrow biopsy specimens: improved detection with use of B-catenin. Am J Clin Pathol 131:49-57

17. Rodeghiero F, Stasi R, Gernsheimer T, Provan D, Arnold DM, Bussel JB, Cines DB, Chong BH, Cooper N, Godeau B, Lechner K, Mazzucconi MG, McMillan R, Sanz MA, Imbach P, Blanchette V, Kühne T, Ruggeri M, George JN (2009) Standardization of terminology, definitions and outcome criteria in immune thrombocytopenic purpura of adults and children: report from an international working group. Blood 113:2386-2393 